\title{
AN EXPANSION RELATED TO THE GENTRAL LIMIT THEOREM
}

\author{
C. R. HEATHCOTE
}

(Received 23 February 1968)

\section{Statement of the problem}

Let $X_{1}, X_{2}, \cdots$ be independent and identically distributed non-lattice random variables with mean zero, variance $\sigma^{2}<\infty$, and partial sums $S_{n}=X_{1}+X_{2}+\cdots+X_{n}$. When a moment $E|X|^{r}, r \geqq 3$, is finite we have (under a minor restriction) the estimate as $n \rightarrow \infty$,

$$
P\left(S_{n} \leqq x \sigma \sqrt{ } n\right)-\int_{-\infty}^{x} \phi(y) d y=\phi(x) \sum_{k=3}^{r} n^{-\frac{1}{2} k+1} R_{k}(x)+o\left(n^{-\frac{1}{2} r+1}\right),
$$

where

$$
\phi(y)=\frac{1}{\sqrt{2 \pi}} e^{-\frac{1}{2} y^{2}},
$$

([1] page 81; [2] page 515; [3] page 220). The $R_{k}(x)$ are polynomials conveniently written in terms of the Hermite polynomials $H_{j}(x)$;

$$
\phi(x) H_{j}(x)=(-1)^{j} \phi^{(j)}(x) .
$$

If $Y$ is a standard normal variable, that is

$$
P(Y \leqq x)=\int_{-\infty}^{x} \varphi(y) d y,
$$

the expansion (1) estimates the difference between the distributions of $S_{n}$ and $Y \sigma \sqrt{ } n$, for large $n$. In this paper we are interested in estimating the difference

$$
E b\left(S_{n}\right)-E b(Y \sigma \sqrt{ } n)
$$

for large values of $n$ and suitable functions $b(x)$. The main result is Theorem 1 given in $\S \mathbf{3}$ and extensions and applications are considered in subsequent sections. The method used relies heavily on the classical approach as expounded in the works cited above by Cramér, Gnedenko and Kolmogorov, and Feller.

We confine our attention to functions $b(x)$ which are bounded and 
integrable. A different technique is required when $b(x)$ grows unboundly with $x$ and this case is not discussed here.

Notation: The symbol $Y$ will always denote a standard normal variable, that is, the density function of $Y$ is

$$
\phi(y)=\frac{1}{\sqrt{2 \pi}} e^{-\frac{1}{2} y^{2}}, \quad-\infty<y<\infty,
$$

with distribution function $P(Y \leqq y)=\Phi(y)$. The characteristic function of $X$ is written

$$
E e^{i \xi X}=\psi(\xi) .
$$

The $j$ th moment of $X$ (when it exists) is $\mu_{j}=E X^{j} . H_{j}(x), j=1,2, \cdots$, denotes the Hermite polynomial defined in (2). We recall that

$$
H_{k+1}(x)=-k H_{k-1}(x)+x H_{k}(x)
$$

and $H_{k}^{\prime}(x)=k H_{k-1}(x)$ so that

$$
\begin{aligned}
H_{k}(0) & =\frac{1}{k+1} H_{k+1}^{\prime}(0)=(-1)^{k / 2}(k-1)(k-3) \cdots 3.1, & & k \text { even } \\
& =0, & & k \text { odd. }
\end{aligned}
$$

Assuming $\mu_{3}, \mu_{4}$ finite the Edgeworth expansion for $P\left(S_{n} \leqq \sigma \sqrt{n x}\right)$ is

$$
P\left(S_{n} \leqq \sigma \sqrt{n} x\right)=\int_{-\infty}^{x} \phi(y)\left\{1+n^{-\frac{1}{2}} P_{3}(y)+n^{-1} P_{4}(y)\right\} d y+o\left(n^{-1}\right)
$$

(e.g. [2] page 508) where

$$
\begin{aligned}
& P_{3}(x)=\mu_{3}\left(6 \sigma^{3}\right)^{-1} H_{3}(x) \\
& P_{4}(x)=\left(\mu_{4}-3 \sigma^{4}\right)\left(24 \sigma^{4}\right)^{-1} H_{4}(x)+\mu_{3}^{2}\left(72 \sigma^{6}\right)^{-1} H_{6}(x)^{1}
\end{aligned}
$$

\section{Preliminaries}

Firstly it should be pointed out that some restrictions must be placed on the function $b(x)$. For example suppose $E|X|^{k}=\infty$ for some $k \geqq \mathbf{4}$ and $b(x)=x^{k}$. Then $E b\left(S_{n}\right)=\int x^{k} d P\left(S_{n} \leqq x\right)$ is either infinite or not defined whereas

$$
E b(Y \sigma \sqrt{n})=\frac{1}{\sigma \sqrt{n} \sqrt{2 \pi}} \int x^{k} e^{-\frac{1}{2}\left(x^{2} / \sigma^{2} n\right)} d x
$$

is certainly defined for all finite $n$. On the other hand if $b(x)$ is continuous and bounded a result of Helly ([7] page 52) shows that

$$
E b\left(S_{n}\right)-E b(Y \sigma \sqrt{ } n) \rightarrow 0
$$

1 Equation (2.13) page 508 of [2], contains a misprint. 
as $n \rightarrow \infty$, in which case it is meaningful to ask for an asymptotic expansion in decreasing powers of $n$. It turns out that the problem can be properly formulated by requiring (i) sufficiently strong regularity conditions on $b(x)$, or (ii) keeping $b(x)$ as general as possible and imposing smoothness conditions on the distribution function of $X$. The hypotheses of Theorem 1 below cannot be claimed to be the most general conditions for the required expansion (9) to hold, but they do cover a reasonably wide variety of cases.

Before proceeding to the Theorem we dispose of a special case that may on occasion prove useful. Suppose that $b(x)$ is a square integrable function admitting an expansion in Hermite polynomials such that

$$
b(x \sigma \sqrt{ } n)=\sum_{k=0}^{\infty} \frac{\beta_{k, n}}{k !} H_{k}(x) .
$$

Then if $S_{n} /(\sigma \sqrt{ } n)$ has a density $f_{n}(x)$ which can be expanded in a convergent Edgeworth type series

$$
f_{n}(x)=\phi(x)\left\{1+\sum_{k=0}^{\infty} c_{k, n} H_{k}(x)\right\}
$$

we have formally

$$
\begin{aligned}
E b\left(S_{n}\right) & =\int b(y \sigma \sqrt{ } n) f_{n}(y) d y \\
& =\sum_{k=0}^{\infty} \frac{\beta_{k, n}}{k !} \int h_{k}(x) \phi(x)\left\{1+\sum_{j=0}^{\infty} c_{j, n} H_{j}(x)\right\} d x \\
& =\beta_{0, n}+\sum_{k=0}^{\infty} \beta_{k, n} c_{k, n} .
\end{aligned}
$$

Hence

where

$$
\begin{aligned}
E b\left(S_{n}\right)-E b(Y \sigma \sqrt{ } n) & =\beta_{0, n}+\sum_{k=0}^{\infty} \beta_{k, n} c_{k, n}-\sum_{k=0}^{n} \beta_{k, n} \int H_{k}(x) \phi(x) d x \\
& =\sum_{k=0}^{\infty} \beta_{k, n} c_{k, n}=\beta_{n}^{\prime} c_{n},
\end{aligned}
$$

$$
\begin{aligned}
& \boldsymbol{\beta}_{n}^{\prime}=\left(\beta_{0, n}, \beta_{1, n}, \beta_{2, n}, \cdots\right) \\
& \boldsymbol{c}_{n}^{\prime}=\left(c_{0, n}, c_{1, n}, c_{2, n}, \cdots\right) .
\end{aligned}
$$

Thus the required difference can be written as the inner product of the coefficient vectors of the Hermite expansions of $b(x \sigma \sqrt{ } n)$ and $f_{n}(x)$. The major drawback to this approach is that even if the vectors $\beta_{n}$ and $\boldsymbol{c}_{n}$ are known, the $c_{k, n}$ at least are complicated functions of $n$ so that rate of convergence properties are in general not readily obtainable from $\boldsymbol{\beta}_{n}^{\prime} \boldsymbol{c}_{n}$. Mainly for this reason we do not use the Hermite expansion for $b(x)$ (even if it exists), but we do assume that $b(x)$ is at least integrable and bounded. 
However we do use the Edgeworth expansion for the distribution of $S_{n} /(\sigma \sqrt{ } n)$. Since the latter can be rearranged as a series in Hermite polynomials the following result will prove useful.

Lemma. If $m$ is a fixed nonnegative integer and $x^{m} b(x) \in L_{\mathbf{1}}$ and is bounded then for $k=1,2, \cdots$.

(7) $\int b(y \sigma \sqrt{n}) \phi(y) H_{k}(y) d y=\frac{1}{\sqrt{2 \pi}} \sum_{j=0}^{m} \frac{(-1)^{j} H_{k+j}(0)}{j ! \sigma^{j+1} n^{\frac{1}{2}(j+1)}} \int x^{j} b(x) d x+o\left(n^{-\frac{1}{2}(m+1)}\right)$,

Proof. Using the Taylor expansion

$$
n \rightarrow \infty \text {. }
$$

$$
\begin{aligned}
\phi(z) H_{k}(z) & =(-1)^{k} \phi^{(k)}(z) \\
& =(-1)^{k}\left\{\sum_{j=0}^{m-1} \frac{z^{j} \phi^{(k+j)}(0)}{j !}+\frac{z^{m} \phi^{(k+m)}(\theta)}{m !}\right\}, \quad|\theta|<1,
\end{aligned}
$$

we find, after a change of variable,

$$
\begin{aligned}
\int b(y \sigma \sqrt{ } n) \phi(y) H_{k}(y) d y & =(-1)^{k} \sum_{j=0}^{m-1} \frac{\phi^{(k+j)}(0)}{(\sigma \sqrt{ } n)^{j+1} j !} \int x^{j} b(x) d x \\
+ & \frac{(-1)^{k}}{(\sigma \sqrt{ } n)^{m+1} m !} \int x^{m} b(x) \phi^{(k+m)}\left(\theta_{n} x \sigma^{-1} n^{-\frac{1}{2}}\right) d x .
\end{aligned}
$$

But $\phi^{(k+m)}\left(\theta_{n} x \sigma^{-1} n^{-\frac{1}{2}}\right)$ is uniformly bounded so that by dominated convergence

$$
\lim _{n \rightarrow \infty} \int x^{m} b(x) \phi^{(k+m)}\left(\theta_{n} x \sigma^{-1} n^{-\frac{1}{2}}\right) d x=\phi^{(k+m)}(0) \int x^{m} b(x) d x .
$$

The assertion of the lemma follows by substituting in $(8)$.

$$
\phi^{(k+j)}(0)=(-1)^{k+j} \phi(0) H_{k+j}(0)
$$

It is useful to recall that $H_{l}(0)=0$ if $l$ is odd and non-zero for $l$ even. Thus the sum on the right hand side of $(7)$ consists of only $1+[m / 2]$ terms.

\section{The main results}

THEOREM 1. If

(i) $b(x)$ and $x b(x)$ are bounded and $L_{1}$,

(ii) $\mu_{4}$ exists and Cramér's condition, $\lim \sup |\psi(\xi)|<1$, holds, then either one of $|\xi| \rightarrow \infty$

(a) $[\psi(\xi)]^{\nu} \in L_{1}$ for some $\nu \geqq 1$ or

(b) $\beta(\xi)=\int e^{i \xi x} b(x) d x \in L_{1}$

ensures that as $n \rightarrow \infty$ 
(9) $\quad E b\left(S_{n}\right)-E b(Y \sigma \sqrt{n})=\frac{1}{\sigma^{2} \sqrt{2 \pi} n^{\frac{3}{2}}}\left\{P_{3}^{\prime}(0) \int x b(x) d x+\sigma P_{4}(0) \int b(x) d x\right\}$

where

$$
+o\left(n^{\left.-\frac{3}{2}\right)}\right. \text {, }
$$

$$
\begin{aligned}
& P_{3}^{\prime}(0)=-\mu_{3} /\left(2 \sigma^{3}\right) \\
& P_{4}(0)=\left(\mu_{4}-3 \sigma^{4}\right)\left(8 \sigma^{4}\right)^{-1}-5 \mu_{3}^{2}\left(24 \sigma^{6}\right)^{-1} .
\end{aligned}
$$

Proof. Put

$$
G_{4, n}(y)=P\left(S_{n} \sigma^{-1} n^{-\frac{1}{2}} \leqq y\right)-\int_{-\infty}^{y} \phi(u)\left[1+n^{-\frac{1}{2}} P_{3}(u)+n^{-1} P_{4}(u)\right] d u,
$$

where $P_{3}(x), P_{4}(x)$ are defined in (6). Then

$$
\begin{aligned}
E b\left(S_{n}\right)-E b(Y \sigma \sqrt{ } n)=\int b(y \sigma \sqrt{ } n) d P\left(S_{n} \sigma^{-1} n^{-\frac{1}{2}} \leqq y\right)-\int b(y \sigma \sqrt{ } n) \phi(y) d y \\
=\int b(y \sigma \sqrt{ } n) d G_{4, n}(y)+\int b(y \sigma \sqrt{ } n)\left[n^{-\frac{1}{2}} P_{3}(y)+n^{-1} P_{4}(y)\right] \phi(y) d y .
\end{aligned}
$$

We show first that if either (a) or (b) holds then the first integral on the right is $o\left(n^{-\frac{3}{2}}\right)$. If (a) is true then $S_{n} \sigma^{-1} n^{-\frac{1}{2}}$ has a density for $n>v$ so that

$$
g_{4, n}(x)=G_{4, n}^{\prime}(x)
$$

exists. It follows that $g_{4, n}(x)=o\left(n^{-1}\right)$ uniformly in $x$ (e.g. [2] page 508) so that

$$
\begin{aligned}
\left|\int b(y \sigma \sqrt{ } n) d G_{4, n}(y)\right| & \leqq o\left(n^{-1}\right) \int|b(y \sigma \sqrt{ } n)| d y \\
& =o\left(n^{-1}\right) \sigma^{-1} n^{-\frac{1}{2}} \int|b(x)| d x=o\left(n^{-\frac{3}{2}}\right) .
\end{aligned}
$$

On the other hand if (b) is true then

$$
\begin{aligned}
\int b(y \sigma \sqrt{n}) d G_{4, n}(y) & =\frac{1}{2 \pi} \iint e^{-i \xi \sigma \sqrt{ } \bar{n} y} \beta(\xi) d \xi d G_{4, n}(y) \\
& =\frac{1}{2 \pi} \int \beta(\xi) \gamma_{4, n}(-\xi \sigma \sqrt{ } n) d \xi \\
& =-(2 \pi \sigma \sqrt{ } n)^{-1} \int \beta\left(-x \sigma^{-1} n^{-\frac{1}{2}}\right) \gamma_{4, n}(x) d x,
\end{aligned}
$$

where

$$
\begin{aligned}
\gamma_{4, n}(\xi) & =\int e^{i \xi x} d G_{4, n}(x), \\
& =\left[\psi\left(\xi \sigma^{-1} n^{-\frac{1}{2}}\right)\right]^{n}-e^{-\left(\frac{1}{2}\right) \xi^{2}} p(\xi),
\end{aligned}
$$

$p(\xi)$ being a polynomial of degree six.

A result in [3] (Theorem $\mathbf{l}$ (b) page 204) states that there is a constant $a$ such that (in our notation) 


$$
\left|\gamma_{4, n}(\xi)\right| \leqq \frac{o(1)}{n}\left(|\xi|^{4}+|\xi|^{6}\right) e^{-(1) \xi^{2}}, \quad|\xi|<a \sqrt{ } n
$$

Hence

$$
\begin{aligned}
\left|n^{-\frac{1}{2}} \int_{|x| \leqq a \sqrt{ } n} \beta\left(-x \sigma^{-1} n^{-\frac{1}{2}}\right) \gamma_{\mathbf{4}, n}(x) d x\right| \\
\quad<\frac{o(1)}{n^{\frac{3}{2}}} \int_{|x|<a \sqrt{ } n}\left|\beta\left(\frac{-x}{\sigma \sqrt{ } n}\right)\right|\left(|x|^{\mathbf{4}}+|x|^{6}\right) e^{-\left(\frac{1}{4}\right) x^{2}} d x=o\left(n^{-\frac{3}{2}}\right),
\end{aligned}
$$

since $\left|\beta\left(-x^{-1} n^{-\frac{1}{2}}\right)\right|$ is bounded by $\int|b(y)| d y$ and $\left(|x|^{4}+|x|^{6}\right) e^{-\left(\frac{1}{4}\right) x^{2}}$ is integrable. It follows that the integral in $(10)$ is $o\left(n^{-\frac{3}{2}}\right)$ if it can be shown that

$$
\frac{n^{\frac{3}{2}}}{2 \pi \sigma \sqrt{ } n} \int_{|x|>a \sqrt{ } n} \beta\left(-x \sigma^{-1} n^{-\frac{1}{2}}\right) \gamma_{4, n}(x) d x \rightarrow 0 .
$$

But this is easily seen to be so since Cramerr's condition implies that the tail integral decreases exponentially fast. Thus supposing without loss of generality that

we have

$$
|\psi(\xi)| \leqq \delta<1 \quad \text { for } \quad|\xi|>a \sigma^{-1}
$$

$$
\begin{aligned}
& \left|\frac{n^{\frac{3}{2}}}{2 \pi \sigma \sqrt{ } n} \int_{|x|>a \sqrt{ } n} \beta\left(-x \sigma^{-1} n^{-\frac{1}{2}}\right) \gamma_{4, n}(x) d x\right| \\
& \quad=\left|\frac{n^{\frac{3}{2}}}{2 \pi} \int_{|y|>a \sigma^{-1}} \beta(-y)\left\{[\psi(y)]^{n}-e^{-\left(\frac{1}{2}\right) y^{2} \sigma^{2} n} p(y \sigma \sqrt{ } n)\right\} d y\right| \\
& \quad \leqq \frac{n^{\frac{3}{2}}}{2 \pi}\left\{\delta^{n} \int_{|y|>a \sigma^{-1}}|\beta(-y)| d y+e^{-\left(\frac{1}{4}\right) a^{2} n} \int_{|y|>a \sigma^{-1}} e^{-\left(\frac{1}{4}\right) v^{2} \sigma^{2} n}|\beta(-y) p(y \sigma \sqrt{ } n)| d y\right\} \\
& \quad \rightarrow 0 .
\end{aligned}
$$

The proof is complete if

$$
\begin{aligned}
\int b(y \sigma \sqrt{ } n)\left[n-\frac{1}{2} P_{3}(y)\right. & \left.+n^{-1} P_{4}(y)\right] \phi(y) d y \\
= & \frac{1}{\sigma^{2} \sqrt{2 \pi} n^{\frac{3}{2}}}\left\{P_{3}^{\prime}(0) \int x b(x) d x+\sigma P_{4}(0) \int b(x) d x\right\}+o\left(n^{-\frac{3}{2}}\right)
\end{aligned}
$$

and that this is the case follows from the Lemma and the definition (6) of $P_{3}(x), P_{4}(x)$.

Note that the condition lim sup $|\psi(\xi)|<1$ excludes from consideration amongst non-lattice variables only a relatively unusual class of purely singular variables. ${ }^{2}$ For lattice variables this condition is of course not satisfied, and the coefficients of the expansion differ from those in (9).

It will frequently be the case that

${ }^{2}$ For an example see [7] page 27. 


$$
E b(Y \sigma \sqrt{ } n)=\frac{1}{\sigma \sqrt{ } n \sqrt{2 \pi}} \int b(x) e^{-\left(x^{2} / 2 \sigma^{2} n\right)} d x
$$

can be evaluated without difficulty. On the other hand an asymptotic expansion for $E b\left(S_{n}\right)$ commencing with a term of order $0\left(n^{-\frac{1}{2}}\right)$ can be readily obtained from

$$
E b(Y \sigma \sqrt{ } n)=\frac{1}{\sigma \sqrt{ } 2 \pi \sqrt{ } n}\left\{\int b(x) d x-\frac{1}{2 \sigma^{2} n} \int x^{2} b(x) d x\right\}+o\left(n^{-\frac{3}{2}}\right) .
$$

Hence

COROLLARY TO THEOREM 1. If in addition to the conditions of the Theorem, $x^{2} b(x)$ is bounded and integrable then

$$
\begin{aligned}
& E b\left(S_{n}\right)=\frac{1}{n^{\frac{1}{2}} \sigma \sqrt{ } 2 \pi} \int b(x) d x+\frac{1}{n^{\frac{3}{2}} \sigma \sqrt{2 \pi}} \\
& \cdot\left\{\sigma P_{4}(0) \int b(x) d x+P_{3}^{\prime}(0) \int x b(x) d x-\frac{1}{2 \sigma} \int x^{2} b(x) d x\right\}+o\left(n^{-\frac{3}{2}}\right) .
\end{aligned}
$$

One of the results of Rosen ([8], Theorem $\mathbf{l}(\mathrm{c})$ ) is that for the partial sums of any independent and identically distributed variables the inequality

$$
P\left(c_{1}<S_{n} \leqq c_{2}\right) \leqq c n^{-\frac{1}{2}}
$$

holds. Taking $b(x)$ as unity for $c_{1}<x \leqq c_{2}$ and zero elsewhere in (11) we obtain a sharpening of Rosén's result for the class of random variables satisfying the conditions of the theorem.

If higher moments exist the right hand side of (9) can be expanded further. In place of $G_{4, n}(y)$ introduced in the proof of theorem 1 we use

$$
G_{r, n}(y)=P\left(S_{n} \leqq y \sigma \sqrt{ } n\right)-\int_{-\infty}^{y} \phi(u)\left[1+\sum_{k=3}^{r} \frac{P_{k}(u)}{n^{\left(\frac{1}{2}\right) k-1}}\right] d u
$$

and similar considerations apply. The $P_{k}(u)$ appearing on the right hand side are the same as those in [2] pages 508-509. We only state the result;

THEOREM 2. Suppose each of $b(x), x b(x), \cdots, x^{m} b(x)$ are bounded and $L_{1}$ and $E|X|^{r}<\infty$, where $r, m$ are fixed integers $r>4, m>1$. If Cramér's condition and either of (a) or (b) of Theorem 1 hold then

$$
E b\left(S_{n}\right)-E b(Y \sigma \sqrt{n})=\sum_{k=3}^{r+m-1} \frac{c_{k}}{n^{k / 2}}+o\left(n^{-\frac{1}{2}(r+m-1)}\right) .
$$

The $c_{k}$ depend only on the first $r$ moments of $X$ and the integrals

$$
\int x^{j} b(x) d x, \quad j=0,1, \cdots, m .
$$


Calculation of the constants $c_{k}$ in (12) is tedious but the method is precisely that used in the proof of Theorem 1.

Extensions are possible in several directions. Firstly the case of independent but non-identically distributed variables can be treated with little additional difficulty provided certain regularity conditions are satisfied. Secondly one can consider a sequence of functions $b_{n}(x)$, which if uniformly bounded and convergent to $b(x)$, yields precisely (9).

\section{Variables with moment generating functions and non-zero mean}

Let $W_{1}, W_{2}, \cdots$ be proper two sided variables independently and identically distributed as $W$. It is assumed that $E W \neq 0$ and that the moment generating function

$$
E e^{-s W}=\theta(s)
$$

exists for real $s$ in some nondegenerate interval $I$. For each $u \in I$ a random variable $W(u)$ can be defined by

$$
P(W(u) \leqq x)=[\theta(u)]^{-1} \int_{-\infty}^{x} e^{-u y} d P(W \leqq y) .
$$

$W(u)$ is said to be conjugate to $W$ with respect to the parameter $u$.

Conjugate variables have proved useful in certain problems related to the central limit theorem (particularly large deviations) and recent expositions are to be found in [5] and [2] (where the term associated is used). The expansions obtained below are extensions of some of the results in $[4]$.

If $Z_{n}=W_{1}+W_{2}+\cdots+W_{n}$, a conjugate variable is

and

$$
Z_{n}(u)=W_{1}(u)+W_{2}(u)+\cdots+W_{n}(u),
$$

Conversely

$$
P\left(Z_{n}(u) \leqq x\right)=\left[\theta(u)^{-n}\right] \int_{-\infty}^{x} e^{-u y} d P\left(Z_{n} \leqq y\right)
$$

$$
P\left(Z_{n} \leqq x\right)=[\theta(u)]^{n} \int_{-\infty}^{x} e^{u y} d P\left(Z_{n}(u) \leqq y\right) .
$$

Thus for a suitable function $a(x)$,

$$
E a\left(Z_{n}\right)=[\theta(u)]^{n} \int a(x) e^{u x} d P\left(Z_{n}(u) \leqq x\right) .
$$

By choosing $u \in I$ in a particular way we use (14) to estimate the difference

$$
E a\left(Z_{n}\right)-E a(Y \sigma \sqrt{ } n),
$$

where as before $Y$ is a standard normal variable. It turns out that optimum results are obtained when $u=s_{0}$, where 


$$
\theta\left(s_{0}\right)=\inf _{s \in I} \theta(s)
$$

That $s_{0}$ is finite for two sided variables is a result of [6], Lemma 2.

Definition. The random variable $W$ is said to satisfy condition $\mathscr{C}$ if

(i) $E W \neq 0$ and $E e^{-s W}=\theta(s)$ exists for $-c_{1}<s<c_{2}$ with $c_{1} \geqq 0$, $c_{2} \geqq 0, c_{1}+c_{2}>0$,

(ii) $\theta\left(s_{0}\right)=\inf _{s} \theta(s)$ is attained for an interior point $s_{0}$, i.e. $-c_{1}<s_{0}<c_{2}$,

(iii) the conjugate variable $W\left(s_{0}\right)$ satisfies Cramér's condition

$$
\limsup _{|\xi| \rightarrow \infty}\left|E e^{i \xi W\left(s_{0}\right)}\right|=\limsup _{|\xi| \rightarrow \infty} \frac{\left|\theta\left(s_{0}-i \xi\right)\right|}{\theta\left(s_{0}\right)}<1 .
$$

Since $\theta^{\prime}(0)=-E W, \mathscr{C}$ (i) and $\mathscr{C}$ (ii) ensure that $s_{0} \neq 0, \theta\left(s_{0}\right)<1$ and $\theta^{\prime}\left(s_{0}\right)=0$. Thus $W\left(s_{0}\right)$ has zero mean and furthermore (since $s_{0}$ is an interior point) has itself a moment generating function, namely

$$
E e^{-\alpha W\left(s_{0}\right)}=\theta\left(s_{0}+\alpha\right) / \theta\left(s_{0}\right),
$$

and so has finite moments of all orders. The results obtained so far are therefore immediately applicable to the sequence $\left\{\left(Z_{n}\left(s_{0}\right)\right\}\right.$, provided $W$ satisfies $\mathscr{C}$, and we use this fact in conjunction with (14) to study $\operatorname{Ea}\left(Z_{n}\right)$.

THEOREM 3. Let $Z_{n}$ be the nth partial sum of independent variables distributed as $W$, where $W$ satisfies condition $\mathscr{C}$. If a $(x)$ is a given function such that $x a(x) e^{s_{0} x}$ is bounded and in $L_{1}$, then as $n \rightarrow \infty$

$$
\begin{aligned}
\frac{E a\left(Z_{n}\right)}{\left[\theta\left(s_{0}\right)\right]^{n}}= & E\left[a\left(Y \sigma_{0} \sqrt{ } n\right) e^{Y s_{0} \sigma_{0} \sqrt{ } n}\right]+\frac{1}{\sigma_{0}^{2} \sqrt{2 \pi} n^{\frac{3}{2}}} \\
& \cdot\left\{\left\{P_{\mathbf{3}, 0}^{\prime}(0) \int x a(x) e^{s_{0} x} d x+\sigma_{0} P_{4,0}(0) \int a(x) e^{s_{0} x} d x\right\}+o\left(n^{-\frac{3}{2}}\right),\right.
\end{aligned}
$$

where

$$
\begin{aligned}
P_{3,0}^{\prime}(0) & =\frac{\theta^{(3)}\left(s_{0}\right)\left[\theta\left(s_{0}\right)\right]^{\frac{1}{2}}}{2\left[\theta^{\prime \prime}\left(s_{0}\right)\right]^{\frac{3}{2}}} \\
P_{4,0}(0) & =\frac{\theta\left(s_{0}\right) \theta^{(4)}\left(s_{0}\right)-3\left[\theta^{(2)}\left(s_{0}\right)\right]^{2}}{8\left[\theta^{(2)}\left(s_{0}\right)\right]^{2}}-\frac{5\left[\theta\left(s_{0}\right)\right]^{4}\left[\theta^{(3)}\left(s_{0}\right)\right]^{2}}{24\left[\theta^{(2)}\left(s_{0}\right)\right]^{3}} \\
\sigma_{0}^{2} & =E\left[W\left(s_{0}\right)\right]^{2}=\theta^{(2)}\left(s_{0}\right) / \theta\left(s_{0}\right) .
\end{aligned}
$$

Proof. From (14) 


$$
\begin{aligned}
& \frac{E a\left(Z_{n}\right)}{\left[\theta\left(s_{0}\right)\right]^{n}}-E\left[a(Y \sigma \sqrt{ } n) e^{s_{0} Y \sigma \sqrt{ } n}\right]=\int a(x) e^{s_{0} x} d P\left(Z_{n}\left(s_{0}\right) \leqq x\right) \\
& \quad-\frac{1}{\sigma \sqrt{ } n \sqrt{2 \pi}} \int a(x) e^{-\frac{1}{2} x^{2}+s_{0} \sigma_{0} \sqrt{ } n x} d x \\
& \quad=\int a\left(\sigma_{0} \sqrt{ } n y\right) e^{s_{0} \sigma_{0} \sqrt{ } n y} d P\left(\frac{Z_{n}\left(s_{0}\right)}{\sigma_{0} \sqrt{ } n} \leqq y\right)-\frac{1}{\sqrt{2 \pi}} \int a\left(\sigma_{0} \sqrt{n} y\right) e^{-\frac{1}{2} \sigma_{0}^{2} n y^{2}+s_{0} \sigma_{0} \sqrt{n} y} d y
\end{aligned}
$$

Writing $Z_{n}\left(s_{0}\right)=S_{n}, b(x)=a(x) e^{\varepsilon_{0} x}$, it is apparent that Theorem $\mathrm{I}$ is directly applicable, and we obtain (15).

The moments in $P_{3}^{\prime}(0), P_{4}(0)$ are now the moments of $W\left(s_{0}\right)$,

$$
E\left[W\left(s_{0}\right)\right]^{k}=(-1)^{k} \frac{\theta^{(k)}\left(s_{0}\right)}{\theta\left(s_{0}\right)} .
$$

An expansion including smaller terms can be similarly derived from Theorem 2.

As an example suppose $a(x)$ is unity for $c_{1}<x<c_{2}$ and zero elsewhere. Then

$$
\begin{aligned}
& E a\left(Z_{n}\right)=P\left(c_{1}<Z_{n} \leqq c_{2}\right) \\
& E\left[a\left(Y \sigma_{0} \sqrt{ } n\right) e^{Y s_{0} \sigma_{0} \sqrt{ } n}\right]=e^{\left(\frac{1}{2}\right) s_{0}^{2} \sigma_{0}^{2} n}\left\{\Phi\left(\frac{c_{2}-s_{0} \sigma_{0}^{2} n}{\sigma_{0} \sqrt{ } n}\right)-\Phi\left(\frac{c_{1}-s_{0} \sigma_{0}^{2} n}{\sigma_{0} \sqrt{ } n}\right)\right\}
\end{aligned}
$$

and (15) yields an exponentially decreasing estimate of $P\left(c_{1}<Z_{n} \leqq c_{2}\right)$.

\section{Application to random walks}

As in the preceding section let $W_{1}, W_{2}, \cdots$ be independent identically distributed variables satisfying the condition $\mathscr{C}$. Let

$$
M_{n}=\max \left(0, Z_{1}, Z_{2}, \cdots, Z_{n}\right), \quad n \geqq 1,
$$

$M_{0}=0$, where as before $Z_{n}$ is the $n$th partial sum of the $W_{i}$. The distribution of $M_{n}$ is in principle obtainable from the Pollaczek-Spitzer formula

$$
\sum_{n=0}^{\infty} z^{n} E e^{i \xi M_{n}}=\exp \left\{\sum_{n=1}^{\infty} z^{n} n^{-1} E e^{i \xi Z_{n}^{+}}\right\}
$$

where $Z_{n}^{+}=\max \left(0, Z_{n}\right)$. If $E W=\mu<0$ it is known that the sequence $\left\{P\left(M_{n} \leqq x\right)\right\}$ converges to a proper distribution function say $P\left(M_{\infty} \leqq x\right)$. We now wish to apply our results to estimating

$$
E\left(M_{\infty}\right)^{k}-E\left(M_{n}\right)^{k}
$$

when $\mu<0$ and $E e^{-s W}=\theta(s)$ exists for at least $-c_{1}<s \leqq 0, c_{1}>0$. Our estimate extends that obtained in $\S$ II.5 of [4] for the difference $E M_{\infty}-E M_{n}$, and also the work of Kingman [6]. 
From (16)

so that

$$
E M_{n}=\sum_{j=1}^{n} j^{-1} E Z_{j}^{+}=\sum_{j=1}^{n} j^{-1} \int_{0}^{\infty} x d P\left(Z_{j} \leqq x\right)
$$

$$
E M_{\infty}-E M_{n}=\sum_{j=n+1}^{\infty} j^{-1} E Z_{j}^{+}
$$

The proof of the following Theorem follows from Theorem 3 by the substitution $a(x)=x^{k}, x>0$, and $a(x)=0$ for $x \leqq 0$.

Theorem 4. If $W$ satisfies $\mathscr{C}$ with $E W<0$ (and hence $s_{0}<0$ ) then for $k=1,2, \cdots$

$$
\begin{aligned}
& \frac{E\left(Z_{n}^{+}\right)^{k}}{\left[\theta\left(s_{0}\right)\right]^{n}}-E\left[\left[\left(Y \sigma_{0} \sqrt{ } n\right)^{+}\right]^{k} e^{Y s_{0} \sigma_{0} \sqrt{ } n}\right] \\
& \quad=\frac{k !}{\left(-s_{0}\right)^{k+2} \sigma_{0}^{2} \sqrt{2 \pi} n^{\frac{3}{2}}}\left\{P_{3,0}^{\prime}(0)(k+1)+\left(-s_{0} \sigma_{0}\right) P_{4,0}(0)\right\}+o\left(n^{-\frac{3}{2}}\right) .
\end{aligned}
$$

To obtain an expansion for $E\left(Z_{n}^{+}\right)^{k}$ itself we must evaluate

$$
\begin{aligned}
E\left[\left\{\left(Y \sigma_{0} \sqrt{ } n\right)^{+}\right\}^{k} e^{Y s_{0} \sigma_{0} \sqrt{ } n}\right] & =\frac{1}{\sigma_{0} \sqrt{n} \sqrt{2 \pi}} \int_{0}^{\infty} x^{k} \exp \left[s_{0} x-\frac{x^{2}}{2 \sigma_{0}^{2} n}\right] d x \\
& =\frac{e^{\frac{1}{2} s_{0}^{2} \sigma_{0}^{2} n}}{\sqrt{2 \pi}} \int_{-s_{0} \sigma_{0} \sqrt{ } n}^{\infty}\left[\sigma_{0} \sqrt{n} y+s_{0} \sigma_{0}^{2} n\right]^{k} e^{-\frac{1}{2} v^{2}} d y
\end{aligned}
$$

and this can be done without difficulty. For example if $k=1$ then

$$
\begin{aligned}
E\left[\left(Y \sigma_{0} \sqrt{ } n\right)^{+} e^{Y s_{0} \sigma_{0} \sqrt{ } n}\right] & =\frac{\sigma_{0} \sqrt{ } n}{\sqrt{2 \pi}}\left\{1+s_{0} \sigma_{0} \sqrt{ } n \sqrt{2 \pi} e^{\frac{1}{2} \frac{8}{0}_{0}^{2} \sigma_{0}^{2} n}\left[1-\Phi\left(-s_{0} \sigma_{0} \sqrt{ } n\right)\right]\right\} \\
& =\frac{1}{\sqrt{2 \pi}}\left\{\frac{1}{s_{0}^{2} \sigma_{0} n^{\frac{1}{2}}}-\frac{3}{s_{0}^{4} \sigma_{0}^{3} n^{\frac{3}{2}}}+\frac{15}{s_{0}^{6} \sigma_{0}^{5} n^{\frac{5}{2}}} \cdots\right\}
\end{aligned}
$$

with $\sigma_{0}^{2}=\theta^{(2)}\left(s_{0}\right) /\left[\theta\left(s_{0}\right)\right]$. Then

$$
E Z_{j}^{+}=\frac{\left[\theta\left(s_{0}\right)\right]^{j}}{\sqrt{2 \pi}}\left\{\frac{1}{s_{0}^{2} \sigma_{0} j^{\frac{1}{2}}}+\frac{1}{s_{0}^{4} \sigma_{0}^{3} j^{\frac{3}{2}}}\left[s_{0}^{2} \sigma_{0}^{2} P_{4,0}(0)-2 s_{0} \sigma_{0} P_{3,0}^{\prime}(0)-3\right]+o\left(n^{-\frac{3}{2}}\right)\right\} .
$$

Hence

$$
\begin{aligned}
E M_{\infty}-E M_{n}= & \sum_{j=n+1}^{\infty} j^{-1} E Z_{j}^{+} \\
= & \frac{1}{s_{0}^{2} \sigma_{0} \sqrt{\overline{2 \pi}}} \sum_{j=n+1}^{\infty} j^{-\frac{3}{2}}\left[\theta\left(s_{0}\right)\right]^{j}+\frac{1}{s_{0}^{4} \sigma_{0}^{3} \sqrt{2 \pi}} \sum_{j=n+1}^{\infty} j^{-\frac{5}{2}}\left[\theta\left(s_{0}\right)\right]^{j} a_{4}\left(s_{0}\right) \\
& +o\left((n+1)^{\left.-\frac{5}{2}\left[\theta\left(s_{0}\right)\right]^{n+1}\right)}\right.
\end{aligned}
$$


which generalises equation II.5.5 of [4]. Somewhat more involved manipulations are required to estimate $E\left(M_{\infty}\right)^{k}-E\left(M_{n}\right)^{k}$ when $k>1$.

\section{References}

[1] H. Cramér, 'Random variables and probability distributions' (Cambridge, 1937).

[2] W. Feller, An introduction to probability theory and its applications' II (Wiley, 1966).

[3] B. V. Gnedenko and A. N. Kolmogorov, 'Limit distributions for sums of independent random variables', trans. K. L. Chung (Addison-Wesley, 1954).

[4] C. R. Heathcote, 'Complete exponential convergence and some related topics', J. Applied Prob., 4, (1967), 217-256.

[5] J. Keilson, 'Green's function methods in probability theory' (Griffin, 1965).

[6] J. F. C. Kingman, 'Some inequalities for the queue GI/G/1', Biometrika, 49, (1962), $315-324$.

[7] E. Lukacs, 'Characteristic functions' (Griffin, 1960).

[8] B. Rosén, 'On the asymptotic distribution of sums of independent identically distributed random variables', Arkiv för Mat., 4, (1963), 323-332.

\section{Australian National University}

Canberra, A.C.T. 